\title{
Nucleotide-binding oligomerization domain 1 (NOD1) modulates liver ischemia reperfusion through the expression adhesion molecules.
}

Guillaume Lassailly,2, Mohamed Bou Saleh ${ }^{1}$, Natascha Leleu-Chavain ${ }^{1,3}$, Massih Ningarhari ${ }^{1,2,}$ Emilie Gantier ${ }^{1}$, Rodolphe Carpentier ${ }^{1}$, Florent Artru ${ }^{1,2}$, Viviane Gnemmi ${ }^{5}$, Benjamin Bertin ${ }^{1}$, Patrice Maboudou ${ }^{6}$, Didier Betbeder ${ }^{1,4}$, Céline Gheeraert ${ }^{7}$, François Maggiotto $^{1}$, Sébastien Dharancy ${ }^{1,2}$, Philippe Mathurin ${ }^{1,2}$, Alexandre Louvet ${ }^{1,2}$, Laurent Dubuquoy $^{1}$

${ }^{1}$ LIRIC - Lille Inflammation Research International Center - U995, Univ. Lille, Inserm, CHU Lille, F-59000 Lille, France.emilie.gantier@inserm.fr, mohamed.bou-saleh@inserm.fr, rodolphe.carpentier@univ-lille2.fr, benjamin.bertin-2@univ-lille2.fr, franzmagg@gmail.com, laurent.dubuquoy@inserm.fr

${ }^{2}$ Service des Maladies de l'Appareil Digestif et de la Nutrition, CHU Lille, F-59000 Lille, France. guillaume.lassailly@chru-lille.fr, m.ningarhari@gmail.com, florent.artru@chrulille.fr, $\quad$ sebastien.dharancy@chru-lille.fr, _ philippe.mathurin@chru-lille.fr, alexandre.louvet@chru-lille.fr

${ }^{3}$ Institut de Chimie Pharmaceutique de Lille, Faculté de Pharmacie, Univ Lille, F-59000 Lille, France.natascha.leleu-2@univ-lille2.fr

${ }^{4}$ Université d'artois, F-62300 Lens, France. betbederdidier985@gmail.com

${ }^{5}$ Service d'anatomopathologie, CHU Lille, F-59000 Lille, France. viviane.gnemmi@chrulille.fr

${ }^{6}$ UF 8832 - Biochimie Automatisée, Pôle de Biologie Pathologie Génétique, CHRU de Lille, F-59000 Lille, France.patrice.maboudou@chru-lille.fr 
${ }^{7}$ U1011 - EGID, Univ. Lille, Inserm, Institut Pasteur de Lille, F-59000 Lille, France. celine.gheeraert@inserm.fr

Key words: Liver ischemia reperfusion; Innate immunity; liver trasnplantation; adhesion molecules; hepatocytes; nanoparticles antagonist, 


\section{Contact Information:}

Laurent Dubuquoy, Ph. D. LIRIC - UMR995, 4 étage Est, Faculté de Médecine - Pôle Recherche, 1 Place Verdun, 59045 Lille Cedex, France. Email: laurent.dubuquoy@inserm.fr; phone : +33 3 20974208, fax: +33 320623525 or Guillaume Lassailly, MD. Service des Maladies de l'Appareil Digestif et de la Nutrition, Hôpital Huriez, CHU Lille, rue Michel Polonovski, 59045 Lille Cedex, France. Email: guillaume.lassailly@chru-lille.fr; phone: +33 3 20445321, fax: +33 320444713

Electronic word count: 7745 (manuscript, figures legend, reference)

List of abbreviations: danger-associated molecular patterns (DAMPs), electron microscopy (EM), extracellular signal-regulated kinase (ERK), intercellular adhesion molecule-1 (ICAM1), ischemia/reperfusion (IR), lactate dehydrogenase (LDH), liver sinusoidal endothelial cells (LSEC), mitogen-activated protein (MAP), nuclear factor kappa B (NF- $\kappa \mathrm{B})$, nucleotidebinding oligomerization domain 1 (NOD1), nanoparticle (np), pathogen-associated molecular patterns (PAMPs), phosphate buffer saline (PBS), paraformaldehyde (PFA), Phenyl Methyl Sulfonyl Fluoride (PMSF), polymorphonuclear neutrophil (PMN), pattern recognition receptors (PRR), Real-time polymerase chain reaction (RT-PCR), relative units (RU), sodium dodecyl sulphate (SDS), Secreted embryonic alkaline phosphatase (SEAP), poly-lactic-coglycolic acid (PLGA), vascular cell adhesion molecule-1 (VCAM-1), wild-type (WT).

Disclosure: The authors declare no conflict of interest in relation to this manuscript.

Financial support: The author acknowledges funding support from the National Institute on Alcohol Abuse and Alcoholism grant 1U01AA021908, AFEF, program StartAIRR ANOD1 Region Haut de France.

Ethics committee number for animal experiments: $n^{\circ} 01757.01$

\section{Author contributions}


E.G., M.B.S., L.D., G.L., F.A., F.M., C.G., P.M. and M.N. generated data, L.D., G.L., S.D. and Ph.M. designed experiments; V.G. performed histological analysis; N.L.C., R.C., B.B., D.B. designed and provided essential tools; L.D., G.L., A.L. analyzed the data; L.D., G.L., N.L.C., R.C. prepared the manuscript; A.L., S.D., F.A. and Ph.M. edited the manuscript; L.D., G.L. and Ph.M. provided experimental funding.

\section{Lay summary}

Nucleotide-binding oligomerization domain 1 (NOD1) is as an important modulator of polymorphonuclear (PMN)-induced liver injury such as in ischemia reperfusion (IR). Here, we show that NOD1 pathway targets liver adhesion molecule expression (ICAM-1 \& VCAM1) on endothelium and on hepatocyte through p38 and ERK signaling pathways. The early increase of adhesion molecule expression after reperfusion, emphasizes the importance of adherence molecules in liver injury severity. In this study we have generated PGLAnanoparticles loaded with NOD1 antagonist. Nanoparticles have shown to reduce liver necrosis, by reducing PMN liver infiltration and adhesion molecules expression. 


\begin{abstract}
(262 words)
Objective: In liver transplantation, organ shortage leads to use marginal grafts that are more susceptible to ischemia reperfusion (IR). We identified nucleotide-binding oligomerization domain 1 (NOD1) as an important modulator of polymorphonuclear (PMN)-induced liver injury such as in IR. Here we aimed at elucidating its role in the liver IR, especially on the endothelium and hepatocyte.
\end{abstract}

Design: NOD1 WT and KO mice were treated with NOD1 agonists and submitted to liver IR. Expression of adhesion molecules were analyzed in total liver, isolated hepatocytes and endothelial cells. Interaction between PMN and hepatocyte was studied in ex vivo co-culture model using electron microscopy (EM) and LDH levels. We generated NOD1 antagonistloaded nanoparticles (np ALINO).

Results: NOD1 agonist treatment increased liver injury, PMN tissue infiltration and upregulated ICAM-1 and VCAM-1 expression 20 hours after reperfusion. NOD1 agonist treatment without IR increased expression of adhesion molecules (ICAM-1, VCAM-1) in total liver and more particularly in WT hepatocytes, but not in NOD1 KO hepatocytes. This induction is dependent of p38 and ERK signaling pathways. As compared to untreated hepatocytes, NOD1 agonist markedly increased hepatocytes lysis in co-culture with PMN as shown by the increase of LDH in supernatants. Interaction between hepatocytes and PMN was confirmed by EM. In a mouse model of liver IR, treatment with np ALINO significantly reduced the area of necrosis, transaminase level and ICAM-1 expression.

Conclusion: NOD1 regulates liver IR injury through induction of adhesion molecules and modulation of hepatocytes-PMN interaction. NOD1 antagonist-loaded nanoparticles reduced liver IR injury and open up new perspectives to prevent IR especially in the context of liver transplantation. 


\section{Introduction}

In the field of liver transplantation, organ shortage is a major issue; especially in western countries where the living donor transplantation is still scare. This situation leaded transplant units to use of liver allografts following donation after cardiac death along with marginal and extended criteria donors ${ }^{1}$. These marginal grafts are known to be more susceptible to early graft dysfunction and retransplantation, increasing morbidity and mortality $^{2-5}$ mostly due to liver ischemia reperfusion (IR) injuries ${ }^{6,7}$. Liver IR is a biphasic phenomenon in which hypoxia-induced lesions are exacerbated after oxygen delivery is restored, shear stress and polymorphonuclear neutrophils (PMN) tissue infiltration ${ }^{8}$. This process of liver injury occurs also during hemodynamic instability and hepatic resection. It is a frequent cause of acute liver dysfunction ${ }^{2-5,9,10}$. Thus, preventing IR and its consequences remains a clinical challenge.

The pathophysiology of liver IR involves numerous cells (i.e. PMN, liver sinusoidal endothelial cells (LSEC) and hepatocytes) and is considered to be an experimental model of PMN-mediated hepatitis ${ }^{3}$. The process of injury occurs during the reperfusion phase with PMN infiltration. The first step of this event is the interaction between PMN and the endothelium, allowing PMN to enter into the liver parenchyma. The upregulation of adhesion molecules (i.e. ICAM-1, VCAM-1, E-Selectin) on LSEC follows reperfusion, allowing PMN recruitment with the rolling process ${ }^{12}$ through the binding of neutrophil adhesion molecules L-Selectin and Mac-1, an heterodimeric integrin $(\mathrm{CD} 11 \mathrm{~b} / \mathrm{CD} 18)^{13}$. The dual role and interaction between endothelium and PMN is accepted to be a cornerstone of the pathophysiology of liver IR. Thus, inhibiting adhesion molecule expression (as ICAM, VCAM, E-Selectin, CD44 and PECAM) or using blocking antibodies have been largely studied to reduce liver injury ${ }^{14}$. 
Contrary to the endothelium, the mechanisms driving the interaction between PMN and hepatocytes during liver IR remain to be clarified. The expression of adhesion molecules by the hepatocyte has been suggested to be important for its interaction with PMN during IR ${ }^{15}$. Intercellular adhesion molecule-1 (ICAM-1) has been shown to be expressed on hepatocyte plasma membrane under IR conditions ${ }^{15}$. However, it remains unclear whether the regulation of ICAM expression on the hepatocyte impacts the severity of IR lesions.

There is evidences that damage-associated molecular patterns (DAMPs) and pathogenassociated molecular patterns (PAMPs) activate PMN ${ }^{16}{ }^{17}$ leading to IR-mediated liver injury. We previously showed that activation of the nucleotide-binding oligomerization domain 1 (NOD1), a cytosolic pattern recognition receptor (PRR), was responsible for activation of PMN function and migration ${ }^{18}$. Indeed, by activating mitogen-activated protein (MAP) kinases such as p38, NOD1 leads to PMN infiltration in the liver, facilitating injury in a model of liver IR. At the opposite, NOD1 knockout (KO) mice were protected against liver IR injury ${ }^{18}$. In addition, NOD1 is significantly expressed in the liver and in hepatocytes ${ }^{19}$, thus NOD1 pathway appears as promising for liver IR injury regulation.

The aim of our study was to dissect the role of the NOD1 pathway on the expression of adhesion molecules in the liver, and more specifically in endothelial cells and in hepatocytes. As IR lesions are induced by PMN liver parenchyma infiltration, we also explored the modulation of hepatocytes and PMN interaction by NOD1. Then, as a proof of concept, we have tested NOD1 as a potential therapeutic target with the use of a NOD1 inhibitor for the prevention of IR lesions in a mouse model. 


\section{Methods}

\section{Reagents}

Three different NOD1 agonists were used in this study. The natural ligand iE-DAP (1 $\mu \mathrm{g} / \mathrm{ml}$ in vitro and $5 \mathrm{mg} / \mathrm{kg}$ in vivo) and its derivative C12-iE-DAP (100 $\mathrm{ng} / \mathrm{ml}$ in vitro and 3.3 $\mathrm{mg} / \mathrm{kg}$ in vivo) were obtained from InvivoGen (Toulouse, France). FK565 (synthetic ligand, $10^{-6} \mathrm{M}$ in vitro and $1 \mathrm{mg} / \mathrm{kg}$ in vivo $)$ was obtained from Dr. M. Chamaillard. TNF $\alpha(50 \mathrm{ng} / \mathrm{ml})$ and IFN $\gamma$ (100ng/ml) were obtained from R\&D (Lille, France). We used ML-130 (Notilinib) and ALINO73 (analog of SB711 ${ }^{20}$ ) synthetized by Natascha Leleu-Chavain as described as NOD1 antagonists ${ }^{20,21}$. The ERK inhibitor (U0126, Sigma, Saint-Quentin Fallavier, France) and p38 inhibitors (SB203580, InvivoGen, Toulouse France) were used at 10 $\mu \mathrm{M}$. Resomer RG503H was used as a PLGA (polylactic-co-glycolic acid) matrix (Evonik Industries AG, Deutschland). (Supplementary CTAT Table)

\section{Animals}

C57BL/6J wild-type mice (Janvier Labs, Le Genest-Saint-Isle, France), Nod1 KO (C57BL/6J background) ${ }^{18}$ and their wild-type littermates (Nod1 WT) were used in this study. Animals were kept in a controlled environment (12 h light/dark cycles) and fed a standard rodent pellet diet (RM1, Special Diet Service, France) ad libitum. Experiments performed on animals were approved by the local ethics committee $\left(n^{\circ} 01757.01\right)$ in accredited facilities ( ${ }^{\circ}$ B59-35010) according to government guidelines ( $\left.{ }^{\circ} 2010 / 63 / U E\right)$.

\section{IR injury}

We used a murine model of $70 \%$ partial hepatic ischemia for $60 \mathrm{~min}$. Mice were or were not treated with a NOD1 agonist $24 \mathrm{~h}$ and $4 \mathrm{~h}$ before surgery. Then mice were anesthetized with $10 \mathrm{mg} / \mathrm{kg}$ xylazine and $100 \mathrm{mg} / \mathrm{kg}$ ketamine, and a midline laparotomy was 
performed. The left lateral and median lobes of the liver were ligatured using Ethicon mersutures F2541 (Ethicon, Issy Les Moulineaux, France). After $60 \mathrm{~min}$ of ischemia, the ligature was removed to initiate hepatic reperfusion. Mice were sacrificed 3hours (for hepatocyte isolation of the ex vivo experiment) or at $\underline{4,8}$ and 20 hours after reperfusion. Blood and liver samples were collected for analysis at each time point. Liver samples were fixed in paraformaldehyde 4\% (w/v in PBS) and paraffin embedded or snap frozen.

\section{Cell isolation and culture}

PMN were isolated following sterile peritonitis as previously described ${ }^{23}$. Briefly, mice received an i.p. injection of $1 \mathrm{~mL}$ of a solution of oyster glycogen at $150 \mu \mathrm{g} / \mu \mathrm{L}$ in phosphate buffer saline (PBS). Sixteen hours later mice were sacrificed and peritoneal cells were harvested using $5 \mathrm{~mL}$ of sterile PBS without $\mathrm{Ca}^{2+} / \mathrm{Mg}^{2+}$. Exudate cells were determined to be $>95 \%$ neutrophils by flow cytometry.

Hepatocytes were isolated by the collagenase perfusion method ${ }^{23}$. Briefly, mice were anesthetized with $2 \%$ isofluran. After laparotomy, the portal vein was cannulated (24G catheter, Terumo, Guyancourt, France). The liver was then perfused for 10 to 20 minutes at 5 $\mathrm{mL} / \mathrm{min}$ with washing buffer (HBSS W/O $\mathrm{Ca}^{2+} \mathrm{Mg}^{2+}$, EDTA $0.5 \mathrm{mM}$, HEPES $50 \mathrm{mM}$ ), then perfused with collagenase buffer (HBSS W/O Ca ${ }^{2+} \mathrm{Mg}^{2+}$, HEPES $50 \mathrm{mM}, \mathrm{CaCl}_{2} 50 \mathrm{mM}$, Collagenase type IV 0.0025\% 850 CDU/mg (C5138, Sigma Aldrich, Saint-Quentin Fallavier, France) for 6 minutes at $5 \mathrm{ml} / \mathrm{min}$.

Following perfusion, the liver was collected in washing buffer and the Glisson's capsule was cut. Cell suspension was filtered in a $70 \mu \mathrm{M}$ cell strainer. Cells were washed twice with washing buffer and centrifuged at $50 \mathrm{~g}$ for 2 minutes at room temperature to pellet murine hepatocytes. Hepatocytes were then washed with hepatocyte culture medium (DMEM Glutamax, glucose $4.5 \mathrm{~g} / \mathrm{L}$, Gentamycin $1 \%$, FCS $10 \%, 10^{-7} \mathrm{M}$ dexamethasone) and 
centrifuged at $500 \mathrm{~g}$ for 5 minutes. One million hepatocytes were plated in 6-well plate (Sarstedt, Marnay, France).

The main challenge to investigate the hepatocyte expression of adhesion molecules was to avoid any contamination of LSEC during the isolation of the mouse primary hepatocytes. To deal with this contingency, we have optimized our experimental method and check the purity of isolated hepatocyte by FACS using CD31 as a marker for endothelial cell contamination. Considered acceptable a purity > 99\% (supplementary figure 1 ). In addition a cytospin followed by cell staining were performed before in vitro and ex vivo analysis to confirm purity.

Primary Human umbilical vein endothelial cells (HUVEC) were obtained from Prof. Eric Boulanger (Lille, France) and were maintained in endothelial cell basal medium supplemented with Bullet Kit (EBM-2) on a culture flask coated with $0.1 \%$ gelatin and maintained at $37^{\circ} \mathrm{C}$ with humidified of $5 \%$ carbon dioxide. HUVEC cultured from passages 4 to 8 were used for the experiments.

\section{Hepatocyte and PMN co-culture}

Freshly isolated hepatocytes were plated on a 48 -well plate $\left(1.25 \times 10^{5}\right.$ cell per well $)$ and incubated at $37^{\circ} \mathrm{C}$ with $5 \% \mathrm{CO}_{2}$ for 36 hours. Purified peritoneal PMN $\left(6.25 \times 10^{5}\right.$ cell per well) were or were not added to the hepatocyte culture for 4 hours in HBSS medium (w/o phenol red). Supernatants were then harvested and centrifuged at $500 \mathrm{~g}$ for $5 \mathrm{~min}$ to discard cells and debris. These supernatants were used to quantify lactate dehydrogenase (LDH) activity using the manufacturer's protocol (Roche, Boulogne-Billancourt, France).

\section{Real-time RT-PCR for mRNA quantification}


Real-time PCR analyses were performed for quantification of mRNA expression. Mouse glyceraldehyde-3-phosphate-dehydrogenase (GAPDH) was used as a housekeeping gene. Sequence-specific PCR primers were designated using Primer3 Software ${ }^{24,25}$; see Table S1 and Supplementary CTAT Table for details). Total RNA was extracted from isolated hepatocytes or liver samples using Nucleospin®RNAII (Macherey-Nagel EURL, Hoerdt, France). First strand cDNA was synthesized from $1 \mu \mathrm{g}$ total RNA using a High Capacity cDNA Reverse Transcription Kit (Applied Biosystems, Courtaboeuf, France). Realtime polymerase chain reaction (RT-PCR) was performed using Power SYBR Green PCR Master Mix (Applied Biosystems, Foster City, CA, USA) in StepOne plus (Applied Biosystems, Foster City, CA, USA).

\section{Western blot}

For western blot studies, proteins were extracted from liver samples or isolated hepatocytes in a lysis buffer including PBS with $1 \%$ Nonidet P40, $0.5 \%$ sodium deoxycholate, 0.1\% sodium dodecyl sulphate (SDS), 100mM Phenyl Methyl Sulfonyl Fluoride (PMSF) and a classical protease inhibitor cocktail (Roche Diagnostics, Penzberg, Germany) as well as phosphatase inhibitors (Sigma-Aldrich, Lyon, France). Fifty micrograms of total proteins were then separated by SDS-PAGE and electroblotted on nitrocellulose membranes. Membranes were incubated overnight with primary antibodies (see Table S2 and Supplementary CTAT Table for details). Immunodetection was completed with a secondary peroxidase-conjugated antibody (1:1000, Dako Laboratories, Trappes, France) and chemiluminescence was performed according to the manufacturer's protocol (ECL, Amersham Pharmacia Biotech, Orsay, France). $\beta$-actin was used as a loading control. Band intensity was analyzed using the MM4000 Pro (CareStream, Noisy-le-Grand, France), 
quantified using Image $\mathbf{J}(\mathrm{NIH})$ and compared to $\beta$-actin. Results were expressed as relative units.

\section{Immunohistochemistry, necrosis area quantification \& $\underline{\text { liver PMN quantification }}$}

Liver samples were fixed in paraformaldehyde (PFA) $4 \%$ and embedded in paraffin. Four-micrometer-thick sections were exposed to primary antibodies after a specific step of heat-performed antigen retrieval (see Table S2 for details) and then to a biotinylated secondary antibody for $1 \mathrm{~h}$. After washing in TBS $+0.05 \%$ Tween 20 , sections were incubated with strepavidin-horseradish peroxidase (Dako Laboratories, Trappes, France). Staining was revealed using 3,3'-diaminobenzidine substrate (Dako Laboratories) for 1 to 5 minutes before the reaction was stopped in distilled water, and counterstained with hematoxylin. Negative controls were incubated with irrelevant serum or isotype-matched immunoglobulin instead of the specific antibody. Stained slides were observed and analyzed under a microscope (Leica, Bensheim, Germany). Pictures were obtained using Axio Scan.Z1 (Zeiss, Göttingen, Germany).

Quantification of necrosis was obtained on digital images of IR livers (3 per mice) obtained from Axio Scan Z1 (Zeiss, Göttingen, Germany). The area of necrosis was defined as the ratio of (area of necrosis/ total liver area) x 100 .

Quantification of PMN infiltration was performed by counting PMN on 8-10 fields of necrosis and/or centrolobular area (magnification, 400) for each mouse.

\section{Human ICAM-1 promoter analysis}

The proximal human ICAM-1 promoter was amplified from human liver genomic DNA using the following primers: forward primer (5'- 
AGGGAGCTCTCGTCAAGATCCAAGCTAGCTG-3')

and

$\left(5^{\prime}-\right.$

GGAAGATCTGTGATCCTTTATAGCGCTAGCC-3') corresponding to fragments between -895 and -57 upstream from ATG. The purified fragment was cloned in the multiple cloning site of the reporter plasmid pGL4.10 [luc2] (Promega, France) using SalI and BglII (NEB, USA). This construct (Prom ICAM, figure 5B) was amplified and purified before sequencing to confirm correct integration of the promoter. CV-1 cells $\left(\mathrm{ATCC}^{\circledR} \mathrm{CCL}-70^{\mathrm{TM}}\right.$ ) were plated in 48-well plates (40000 cells/well) and transfected with empty pGL4.10 [luc2] or Prom ICAM (100 ng/well) and a human NOD1 expression vector (20 ng/well, pHA-NOD1 kind gift from Dana J. Philpott). After 20 hours, cells were washed and lysed for luminescence quantification.

\section{Nanoparticle synthesis, formulation and characterization.}

Poly-Lactic co-glycolic acid (PLGA) nanoparticles (np) were prepared as previously described ${ }^{26}$. The Resomer RG503H (10mg) was dissolved in $1 \mathrm{ml}$ of an organic phase consisting of acetone and ethanol (85:15). After complete dissolution of the Resomer, it was injected into $10 \mathrm{ml}$ water by stirring for 30 minutes at room temperature $\left(21\right.$ to $\left.24{ }^{\circ} \mathrm{C}\right)$. Organic solvents were then evaporated with a rotary vacuum evaporator at $120 \mathrm{rpm}, 28^{\circ} \mathrm{c}$ for 4 minutes. The resulting nanoparticles are used as a control (np). Association with the NOD1 antagonist was performed by post-loading and adding the antagonists into the nanoparticle suspension. ML130 was dissolved in DMSO at $100 \mathrm{mg} / \mathrm{ml}$ and loaded with np from $0.075 \%$ to $3 \%(\mathrm{w} / \mathrm{w})$ for in vitro cell delivery corresponding to treatment from 0.125 to $5 \mu \mathrm{M}(\mathrm{NP}-$ ML130). ALINO73 was dissolved in DMSO at $20 \mathrm{mg} / \mathrm{ml}$ and loaded with $\mathrm{np}$ from $0.2 \%$ to $4 \%(\mathrm{w} / \mathrm{w})$ for in vitro cell delivery corresponding to treatment from 0.25 to $5 \mu \mathrm{M}$ (npALINO). For in vivo delivery, ALINO73 was loaded at 7.5\% (w/w NP) and 5\% (w/v) of glucose was added to the final suspension to compensate for osmolarity. 
The in vivo formulation of np ALINO was characterized for size (Z-Average $=92.8 \mathrm{~nm}$ ) and zeta potential $(-11.8 \mathrm{mV})$ respectively by dynamic light scattering and electrophoretic mobility analysis on a ZetaSizer NanoZS (Malvern Instrument, France). The poly dispersity index (PDI) was 0.259 .

\section{Statistics}

Data were expressed as medians, means \pm SEM or means \pm SD. All comparisons were analyzed using the Mann \& Whitney test. Statistics were calculated using GraphPad Prism version 5.0, (GraphPad Software, San Diego, CA, USA). Differences were considered to be statistically significant if $\mathrm{p}$ was $<0.05$. 


\section{Results}

Induction of adhesion molecule expression in liver of mice treated with NODI agonists, in normal condition (without liver IR)

$\underline{\text { Twenty hours after treatment with NOD1 agonist injection in normal condition }}$ (without liver IR), mouse livers displayed significant induction of proteins ICAM-1 and VCAM-1, two important adhesion molecules (1.4 RU vs. $0.8 \mathrm{RU}, \mathrm{p}=0.032$ and $0.25 \mathrm{RU}$ vs. $\underline{0.00 \mathrm{RU}, \mathrm{p}=0.008 \text { respectively), compared to vehicle-treated mice. There was no effect of }}$ NOD1 agonist on E-selectin and P-selectin protein expression (figure 1A). The immunostaining of ICAM-1 showed that NOD1 agonist treatment increased ICAM-1 expression not only on endothelium but also on hepatocyte membranes compared to untreated $\underline{\text { livers which mainly displayed endothelial staining (figure 1B). }}$

NODI agonist increases liver IR injury and up-regulates ICAM-1 and VCAM-1.

$\underline{\text { Twenty hours after IR, liver of mouse treated with NOD1 agonist displayed more }}$ necrosis area than those treated with vehicle (figure 2A). The kinetic analysis of PMN infiltration in liver parenchyma revealed that regardless NOD1 treatment; there was no PMN infiltration 4 hours after reperfusion. It became significant 8 hours after reperfusion. At 8 and $\underline{20 \text { hours after reperfusion, the NOD1 agonist treatment significantly induced PMN liver }}$ $\underline{\text { infiltration as compared to vehicle treatment. Upon NOD1 agonist treatment } \mathrm{KC} \text { was up }}$ regulated 20 hours after reperfusion. (figure $2 \mathrm{~B}$ )

Four hours after reperfusion, transaminases increased (AST 2078 \pm 350 IU/L $\underline{\text { vs. } 1921 \pm 346.5 \mathrm{IU} / \mathrm{L} \text { and ALT } 2885 \pm 547.7 \mathrm{IU} / \mathrm{L} \text { vs. } 2610 \pm 385 \mathrm{IU} / \mathrm{L}) \text { without differences }}$ between the two treatment conditions (NOD1 ago vs. vehicle). ALT increased at 8 hours of $\underline{\text { reperfusion, and were significantly higher in the NOD1 agonist group }(3910 \pm 25 \text { IU/L vs. }}$ 
$\underline{3139.6 \pm 292.5 \mathrm{IU} / \mathrm{L} ; \mathrm{p}=0.05) \text { whereas AST were not different in each group }(1802.0 \pm 137.3}$ $\underline{\text { IU/L vs. } 1905 \pm 159.6 \mathrm{IU} / \mathrm{L}) \text {. At } 20 \text { hours of reperfusion, the NOD1 agonist conditions }}$ presented significantly higher values of transaminase than vehicle conditions (AST 3173 \pm 96 $\underline{\text { IU/L vs. } 2402 \pm 109 \mathrm{IU} / \mathrm{L} ; \mathrm{p}<0.01 \text { and ALT } 4627 \pm 78 \mathrm{IU} / \mathrm{L} \text { vs.3062 } \pm 52 \mathrm{IU} / \mathrm{L} ; \mathrm{p}<0.01 \text { ) (figure }}$ $\underline{2 \mathrm{C}) .}$

NOD1 agonist treatment up-regulated Icam-1 and Vcam-1 mRNA expression at early time point after reperfusion (4 hours) (figure 2D). This mRNA overexpression began before $\underline{\text { PMN infiltration and the establishment of histological necrosis (Supplementary figure 2). At a }}$ protein level, ICAM-1 and VCAM-1 were significantly induced 20 hours after reperfusion in mice treated with NOD1 agonist (ICAM-1: 1.8 to $2.6 \mathrm{RU} ; \mathrm{p}=0.049$ and VCAM-1: 0.37 to 0.94 RU; $\mathrm{p}=0.049$ ) (figure 2E).

Considering other adhesion molecules, there was no difference of expression after NOD1 agonist treatment in liver IR condition for E-selectin and P-selectin $(\mathrm{p}<0.05)$ (figure $\underline{2 \mathrm{E})}$.

NOD1 pathway targets ICAM-1 and VCAM-1 expression in vitro in primary endothelial cell and hepatocyte

HUVEC were cultured and exposed to NOD1 agonist at different dosage (0, 0.1 and 1 $\mu \mathrm{g})$. The mRNA expression of Icam-1, Vcam-1 and E-selectin were induced by NOD1 agonist treatment in a dose dependent manner. However, Pecam mRNA expression was not significantly modified by NOD1 activation (figure 3A).

Mouse primary hepatocytes were cultured in vitro. Before analysis, the purification of isolated cells was confirmed (hepatocyte purity > 99\%, supplementary figure 1). Primary hepatocytes were then exposed to NOD1 agonists under basal or inflammatory (TNF/IFN) 
conditions. As shown in figure 3B , Icam-1 mRNA was induced twice in Nod1 wild-type (WT) hepatocytes treated with the NOD1 agonist, as well as under inflammatory conditions induced by TNF/IFN. Vcam-1 mRNA was significantly induced by NOD1 activation (1.2 RU vs $3.3 \mathrm{RU}, \mathrm{p}=0.005$, figure $3 \mathrm{~A}$ ). Induction of Icam-1 and Vcam-1 mRNA expression was synergistic with the NOD1 agonist and TNF/IFN treatments (figure 3B). These data were confirmed at the protein level in mouse primary isolated hepatocytes which showed that ICAM-1 and VCAM-1 were significantly increased by the NOD1 agonist both under basal and inflammatory conditions (igure 3D). To confirm the specificity of the NOD1 agonist, hepatocytes were isolated from Nod1 KO mice and exposed to NOD1 agonist treatment under basal and inflammatory conditions. Real-time PCR and Western-blot showed that the NOD1 agonist did not induce ICAM-1 and VCAM-1 in NOD1 KO hepatocytes but responded to TNF/IFN treatment (figure 3C,E).

\section{NOD1 pathway targets ICAM-1 and VCAM-1 expression ex vivo in hepatocyte}

In ex-vivo conditions, NOD1 agonist treatment of sham operated mice induced significant hepatocyte expression of Icam-1 and Vcam-1 mRNA (6-fold and 10-fold increase, respectively) compared to vehicle-treated animals (figure 4A). IR alone also induced Icam-1 and Vcam-1 mRNA (4 fold and 5-fold increase, respectively). NOD1 activation during IR markedly increased hepatocyte Icam-1 and Vcam-1 mRNA expression (25-fold and 20-fold increase, respectively) compared to vehicle-treated sham animals (figure 4A). At the protein level, NOD1 agonist treatment in sham-operated mice strongly induced hepatocyte expression of ICAM-1 and VCAM-1 (7.1-fold and 4.0-fold, respectively, figure 4C) compared to untreated mice. After 3 hours of reperfusion, induction of the ICAM-1 and VCAM-1 proteins also increased significantly (2.1-fold and 2.0-fold respectively, figure 4C). NOD1 agonist 
treated Nod1 WT mice that underwent liver IR showed massive induction of hepatocyte expression of ICAM-1 and VCAM-1 protein (37-fold and 109-fold, respectively) compared to vehicle-treated sham animals. More importantly, NOD1 agonist treatment increased ICAM-1 and VCAM-1 protein expression by 17.7 and 54.5 fold respectively upon liver IR conditions (figure 4C).

In Nod1 KO mice, the NOD1-mediated induction of ICAM-1 and VCAM-1 expression was blunted both following IR or a sham-operation (figure 4B,D).

\section{NOD1 activation in hepatocytes amplifies the interaction with PMN and subsequent lysis}

Examination of the co-culture by electron microscopy revealed important adhesion of PMNs on hepatocytes which showed signs of distress such as swelling of mitochondria, cytoplasm clarification and lysosome (figure 5A).

Hepatocytes isolated from Nod1 WT mice that were or were not treated with a NOD1 agonist showed low levels of spontaneous lysis when cultured alone (figure 5B). A significant increase in LDH activity was observed when hepatocytes from untreated mice were cocultured with PMN isolated from untreated WT mice. When hepatocytes from NOD1 agonisttreated mice were co-cultured with PMN isolated from untreated WT mice, there was a significant increase in LDH activity compared to hepatocytes from vehicle-treated animals (figure 5B).

To confirm the specificity of NOD1 in relation to this event, we reproduced these experiments with hepatocytes isolated from Nod1 KO mice treated or not with a NOD1 specific agonist. As observed in figure 5C, targeting of Nod1 KO hepatocytes by PMN was reduced and NOD1 agonist-induced lysis was completely blunted. 


\section{NOD1 signaling pathways driving ICAM-1 and VCAM-1 expression in hepatocytes}

A liver active form of ERK (phospho ERK) was induced from 10 to $30 \mathrm{~min}$ after treatment with a NOD1 agonist (igure 6A) . A similar pattern was observed for p38 since active phospho p38 was induced at 10 and $30 \mathrm{~min}$ and then returned to the steady state $60 \mathrm{~min}$ after stimulation with a NOD1 agonist (figure 6B). Treatment with the ERK inhibitor (U0126) significantly limited the increase in Icam-1 and significantly blocked the Vcam-1 mRNA overexpression induced by the NOD1 agonist (figure 6C-D). The p38 inhibitor (SB203580) further blocked NOD1-induced hepatocyte Icam-1 and Vcam-1 mRNA expression. Cotreatment with both ERK and p38 inhibitors did not increase blockade of the NOD1 effect.

In vitro, we first cloned the human ICAM-1 promoter (figure 6E) and showed that NOD1 activation by an agonist resulted in a 50\% increase in luciferase activity driven by this promoter compared to untreated cells (figure 6F).

\section{NOD1 antagonist development to prevent IR-induced liver injury}

ML130 and ALINO73 are two specific NOD1 inhibitors that have been identified by screening different libraries of chemical compounds for activation or inhibition of NOD receptors ${ }^{20,21}$. In an in vitro model that allows evaluation of NOD1 activation, cells were treated with a NOD1 agonist and increasing doses of either ML130 or ALINO73 (supplementary figure 3A-B). Treatment with a NOD1 agonist induced significant expression of the reporter gene $\left(\mathrm{SEAP}, \mathrm{OD}_{655} 0.165 \pm 0.014\right.$ ). Addition of ML130 resulted in a dosedependent decrease in NOD1 activity from $0.16 \mu \mathrm{M}\left(\mathrm{OD}_{655} 0.106 \pm 0.014\right)$ to maximum inhibition at $5 \mu \mathrm{M}\left(\mathrm{OD}_{655} 0.013 \pm 0.014\right)$. Treatment with ALINO73 led to dose dependent inhibition of NOD1 activity from $0.16 \mu \mathrm{M}\left(\mathrm{OD}_{655} 0.197 \pm 0.020\right.$ vs $\left.0.140 \pm 0.021\right)$ to maximum inhibition at $2.5 \mu \mathrm{M}\left(\mathrm{OD}_{655} 0.030 \pm 0.014\right)$. 
Because both NOD1 antagonists are not soluble in injectable vehicles used for in vivo applications, ML130 and ALINO73 were associated with PLGA nanoparticles to facilitate in vivo delivery to the liver.

The effect of antagonist-loaded nanoparticles on NOD1 activity was tested in the same in vitro system as above (supplementary figure 3C-D). As shown in supplementary figure $3 \mathrm{C}$ when ML130 was associated with PLGA nanoparticles, it lost its inhibitory potential on NOD1 activity, ALINO73-loaded nanoparticles (np ALINO) appeared to be at least as effective as free ALINO73 for the dose-dependent inhibition of NOD1 activity in vitro (supplementary figure 3D).

\section{Modulating IR-induced liver injury with NOD1 antagonist nanoparticles}

np ALINO was then used to test the in vivo potential of NOD1 antagonists. Mice received an intravenous injection of nanoparticles that was either empty (np Empty) or loaded with the NOD1 inhibitor (np ALINO) before undergoing one hour of ischemia. As shown in figure 7A, mice treated with np Empty displayed large areas of IR-induced necrosis. However, when treated with the np ALINO the area of necrosis was lower in mouse livers $(26.0 \pm 3.2 \%$ vs $15.6 \pm 2.9 \%, \mathrm{p}=0.038)$ (figure $7 \mathrm{~B}-\mathrm{C}$ ). These results were confirmed by determining serum transaminase. After treatment with np ALINO, serum ALT significantly decreased compared to treatment with empty nanoparticles $(3935 \pm 493$ IU/L vs $5814 \pm 615$ IU/L, p=0.040) (figure 7D). This improvement was associated with a lower liver expression of $\underline{\text { KC mRNA and lower infiltration of PMN in liver parenchyma, whereas the PMN in }}$ centrolobular vessels were unchanged (figure 7E-7F). The expression of ICAM-1 in the liver was reduced after treatment with np ALINO, as observed using immunohistochemistry (figure $\underline{7 G) .}$ 


\section{DISCUSSION}

We have demonstrated in this study that NOD1 pathway modulates liver IR injury through the induced expression of adhesion molecules. These adhesion molecules are mainly ICAM-1 and VCAM-1 and are expressed on LSEC as well as on hepatocytes. With an exploratory experiment using NOD1 antagonist-loaded nanoparticles, we strongly suggest that targeting NOD1 in vivo is able to reduce liver IR injury in part by reducing the expression of ICAM-1 in the liver.

NOD1 has been previously shown to impact liver IR through increased CD11b expression in PMN ${ }^{18}$. However, the role of NOD1 pathway in the liver, and more specifically the expression of adhesion molecules on hepatocyte and endothelium was unknown. Numerous studies have demonstrated the clear impact of adhesion molecules, especially on endothelium for the regulation of histological lesions and PMN tissue infiltration ${ }^{14,27,28}$. Our study emphasizes the role of NOD1 on adhesion molecule expression in the pathophysiology of liver IR. Indeed, ICAM-1 and VCAM-1 expression were increased upon NOD1 agonist $\underline{\text { treatment during liver IR. This up-regulation of adhesion molecules in the whole liver and }}$ hepatocyte occurred within the first hours of reperfusion, before necrosis establishment and PMN infiltration. This early expression of adhesion molecules is then probably a part of the first steps of pathophysiological mechanisms at the origin of reperfusion lesions.

At a cellular level, and more precisely in the context of in vitro culture of endothelial cells, Eselectin in addition to ICAM-1 and VCAM-1 is largely expressed and modulated after NOD1 pathway activation. However, the absence of significant induction of E-selectin expression after NOD1 activation at the whole organ level suggests that the endothelium is not the exclusive actor of expression of adhesion molecule in the liver. The ex-vivo and in vitro experiments demonstrate that hepatocytes are able to express ICAM-1 and VCAM-1 and 
these last are inducible upon NOD1 agonist treatment. Other adhesion molecules (CD44, Pselectin, PECAM) have been screened on hepatocytes, but with no significant modulation of their expression by NOD1 agonist (data not shown).

Although it is central, the PMN/hepatocyte interaction during IR has not been as extensively investigated as the PMN/endothelial interaction. During warm/cold ischemia, endothelial tissue is injured ${ }^{29}$, sinusoidal endothelial cells are detached and sloughed into the sinusoid lumen ${ }^{30}$, allowing PMN direct access to the hepatocyte ${ }^{31,32}$ as confirmed by our analysis by electron microscopy (EM) (supplementary figure 4). We then designed a coculture experiment to model the PMN/hepatocyte interface and clarify the hepatocellular role of NOD1 activation in this interaction. EM analysis of our model confirmed the direct interaction between both cell types leading to hepatocyte death. Quantification of LDH shows that activation of NOD1 in the hepatocyte increases hepatocellular death upon PMN binding. The results of the interaction between Nod1 KO hepatocyte and PMN suggest that blocking hepatocyte NOD1 could prevent PMN-induced hepatocyte death in vitro.

In relation to the intracellular pathway, the effect of NOD1 appeared mediated through ERK and p38 phosphorylation in hepatocytes. The regulatory effect of ERK and p38 inhibitors confirms the role of the MAPK pathway in NOD1-mediated ICAM-1 and VCAM-1 expression. These findings support data obtained in other cellular types such as PMN ${ }^{18,19}$.

NOD1 is expressed widely in many organs and cell types ${ }^{33}$. The clinical potential of NOD1 antagonists could be extended to other organs such as renal IR pathogenesis ${ }^{34}$ or myocardium infarct ${ }^{35}$. In the kidney, Nod1 deficient mice are partially protected from IRinduced kidney injury. NOD1 activation by diaminopimelic acid worsens cardiac IR injury in particular through p38 MAPK. Nod1 knockdown using siRNA in cardiomyocytes attenuates cell death. The role of NOD1 in innate immune cells suggests that NOD1 antagonists should 
be evaluated for sepsis ${ }^{36}$ or PMN-related hepatitis (i.e. alcoholic hepatitis) ${ }^{37-39}$. The present results can serve as a basis for future studies investigating the therapeutic potential of NOD1 antagonists.

Our results and previous studies performed in the liver ${ }^{18,} 19$ strongly support evaluating the proof of concept for the use of NOD1 antagonists to prevent liver IR injury. Previous screening of chemical libraries on NOD receptors ${ }^{20}$ led us to choose two prototypic NOD1 inhibitors from 2 different chemical entities based on their optimal IC50: ML130 and ALINO73 with the greatest inhibitory effect (supplementary figure 3). ML130 and ALINO73 are insoluble in aqueous solutions and insufficiently soluble in non-toxic hydrophobic solvents. Because these low hydrophilic properties prevented their use in vivo, we used nanoparticles to transport insoluble NOD1 antagonists to the liver ${ }^{40}$. We choose PLGA nanoparticles because of their high biocompatibility and FDA approved safety. PLGA is currently used in several drugs such as: Sandostatin LAR@ (USAN (United States Adopted Name): Octreotide) ${ }^{41}$ for neuroendocrine tumors, Bydureon $\odot$ (USAN: Exenatide) ${ }^{42}$ in diabetes and Risperdal Consta $\bigodot$ (USAN: Risperidone) in schizophrenia ${ }^{43}$.

The in vitro testing of PLGA nanoparticles loaded with ML130 or ALINO73 showed that np ML130 was not effective while np ALINO inhibited NOD1 activity at least as efficiently as free ALINO73 suggesting a high bioavailability despite the PLGA integration. In mice, this NOD1 antagonist-loaded nanoparticle, np ALINO, decreased the area of necrosis induced by liver IR by about $40 \%$. This decrease in histological lesions was associated with a $33 \%$ decrease in serum transaminase supporting npALINO as an interesting therapeutic approach to prevent liver IR. The npALINO treatment was also associated with lower KC mRNA expression in the liver, lower PMN infiltration and reduced ICAM expression. The 
reduction of $\mathrm{KC}$ expression could have reduced total amount of PMN recruited to the liver. Indeed, npALINO did not impact the number of PMN in centrolobular vessels, but reduced significantly the severity of PMN infiltration in necrosis areas. These results raise the therapeutic potential of NOD1 antagonists in preventing hepatic IR in humans. The use of NOD1 antagonists should be tested during different steps of liver transplantation. These NOD1 antagonists could be use either in the donor before the organs are harvested, or added to the preservation protocol, as well as a treatment during reperfusion in the recipient.

In conclusion, this study states that NOD1 regulates adhesion molecules expression in the $\underline{\text { liver. NOD1 activation and blockage experiments confirm that NOD1 pathway modulates the }}$ severity of liver IR injury. These data complement the impact of NOD1 on PMN function and migration. Thus NOD1 has a dual role and targets two different actors implicated in reperfusion injury. However, the relative contribution of each actor (PMN or adhesion molecule) on liver IR injury is unclear. New experiments are needed to puzzle out the full $\underline{\text { mechanism of NOD1 pathway. This work supports the development of NOD1 inhibitors. }}$

\section{ACKNOWLEDGEMENTS:}

Authors would like to thank Nathalie Jouy, Anne Loyens and Meryem Tardivel from the Flow Core Facility - BioImaging Center Lille (F-59000 Lille, France) for the access and excellent technical support. Thanks to Melanie Legrand, Cyril Degraeve, Amélie Mine, Emeline Gorecki, Justine Beauchamp, Mélanie Bollier, Perrine Six and Arnaud Dendooven for technical assistance. 


\section{REFERENCES}

1. Casavilla A, Ramirez C, Shapiro R, et al. Experience with liver and kidney allografts from non-heart-beating donors. Transplantation 1995;59:197-203.

2. Clavien PA, Harvey PR, Strasberg SM. Preservation and reperfusion injuries in liver allografts. An overview and synthesis of current studies. Transplantation 1992;53:95778.

3. Jaeschke H. Mechanisms of Liver Injury. II. Mechanisms of neutrophil-induced liver cell injury during hepatic ischemia-reperfusion and other acute inflammatory conditions. Am J Physiol Gastrointest Liver Physiol 2006;290:G1083-8.

4. Lentsch AB, Kato A, Yoshidome H, et al. Inflammatory mechanisms and therapeutic strategies for warm hepatic ischemia/reperfusion injury. Hepatology 2000;32:169-73.

5. Serracino-Inglott F, Habib NA, Mathie RT. Hepatic ischemia-reperfusion injury. Am J Surg 2001;181:160-6.

6. Lee DD, Singh A, Burns JM, et al. Early allograft dysfunction in liver transplantation with donation after cardiac death donors results in inferior survival. Liver Transpl 2014;20:1447-53.

7. Merion RM, Pelletier SJ, Goodrich N, et al. Donation after cardiac death as a strategy to increase deceased donor liver availability. Ann Surg 2006;244:555-62.

8. Peralta C, Jimenez-Castro MB, Gracia-Sancho J. Hepatic ischemia and reperfusion injury: effects on the liver sinusoidal milieu. J Hepatol 2013;59:1094-106.

9. Jaeschke H. Molecular mechanisms of hepatic ischemia-reperfusion injury and preconditioning. Am J Physiol Gastrointest Liver Physiol 2003;284:G15-26.

10. Glanemann M, Langrehr JM, Stange BJ, et al. Clinical implications of hepatic preservation injury after adult liver transplantation. Am J Transplant 2003;3:1003-9. 
11. Caldwell-Kenkel JC, Thurman RG, Lemasters JJ. Selective loss of nonparenchymal cell viability after cold ischemic storage of rat livers. Transplantation 1988;45:834-7.

12. Springer TA. Adhesion receptors of the immune system. Nature 1990;346:425-34.

13. Steinhoff G, Behrend M, Schrader B, et al. Intercellular immune adhesion molecules in human liver transplants: overview on expression patterns of leukocyte receptor and ligand molecules. Hepatology 1993;18:440-53.

14. Ala A, Dhillon AP, Hodgson HJ. Role of cell adhesion molecules in leukocyte recruitment in the liver and gut. Int J Exp Pathol 2003;84:1-16.

15. Benkoel L, Dodero F, Hardwigsen J, et al. Expression of intercellular adhesion molecule-1 (ICAM- 1) during ischemia-reperfusion in human liver tissue allograft: image analysis by confocal laser scanning microscopy. Dig Dis Sci 2003;48:2167-72.

16. Lu L, Zhou H, Ni M, et al. Innate Immune Regulations and Liver IschemiaReperfusion Injury. Transplantation 2016;100:2601-2610.

17. Jaeschke H. Reactive oxygen and mechanisms of inflammatory liver injury: Present concepts. J Gastroenterol Hepatol 2011;26 Suppl 1:173-9.

18. Dharancy S, Body-Malapel M, Louvet A, et al. Neutrophil migration during liver injury is under nucleotide-binding oligomerization domain 1 control. Gastroenterology 2010;138:1546-56, 1556 e1-5.

19. Scott MJ, Chen C, Sun Q, et al. Hepatocytes express functional NOD1 and NOD2 receptors: a role for NOD1 in hepatocyte $\mathrm{CC}$ and $\mathrm{CXC}$ chemokine production. $\mathrm{J}$ Hepatol 2010;53:693-701.

20. Rickard DJ, Sehon CA, Kasparcova V, et al. Identification of selective small molecule inhibitors of the nucleotide-binding oligomerization domain 1 (NOD1) signaling pathway. PLoS One 2014;9:e96737. 
21. Khan PM, Correa RG, Divlianska DB, et al. Identification of Inhibitors of NOD1Induced Nuclear Factor-kappaB Activation. ACS Med Chem Lett 2011;2:780-785.

22. Yamashita T, Ishibashi Y, Nagaoka I, et al. Studies of glycogen-induced inflammation of mice. Dynamics of inflammatory responses and influence of antiinflammatory drugs and protease inhibitors. Inflammation 1982;6:87-101.

23. Seglen PO. Preparation of isolated rat liver cells. Methods Cell Biol 1976;13:29-83.

24. Koressaar T, Remm M. Enhancements and modifications of primer design program Primer3. Bioinformatics 2007;23:1289-91.

25. Untergasser A, Cutcutache I, Koressaar T, et al. Primer3--new capabilities and interfaces. Nucleic Acids Res 2012;40:e115.

26. Le Broc-Ryckewaert D, Carpentier R, Lipka E, et al. Development of innovative paclitaxel-loaded small PLGA nanoparticles: study of their antiproliferative activity and their molecular interactions on prostatic cancer cells. Int J Pharm 2013;454:712-9.

27. Eltzschig HK, Eckle T. Ischemia and reperfusion--from mechanism to translation. Nat Med 2011;17:1391-401.

28. Zhai $\mathrm{Y}$, Petrowsky $\mathrm{H}$, Hong $\mathrm{JC}$, et al. Ischaemia-reperfusion injury in liver transplantation--from bench to bedside. Nat Rev Gastroenterol Hepatol 2013;10:7989.

29. Selzner N, Rudiger H, Graf R, et al. Protective strategies against ischemic injury of the liver. Gastroenterology 2003;125:917-36.

30. Gao W, Bentley RC, Madden JF, et al. Apoptosis of sinusoidal endothelial cells is a critical mechanism of preservation injury in rat liver transplantation. Hepatology $1998 ; 27: 1652-60$. 
31. Jaeschke H, Farhood A, Smith CW. Neutrophils contribute to ischemia/reperfusion injury in rat liver in vivo. FASEB J 1990;4:3355-9.

32. Imamura H, Brault A, Huet PM. Effects of extended cold preservation and transplantation on the rat liver microcirculation. Hepatology 1997;25:664-71.

33. Philpott DJ, Sorbara MT, Robertson SJ, et al. NOD proteins: regulators of inflammation in health and disease. Nat Rev Immunol 2014;14:9-23.

34. Shigeoka AA, Kambo A, Mathison JC, et al. Nod1 and nod2 are expressed in human and murine renal tubular epithelial cells and participate in renal ischemia reperfusion injury. J Immunol 2010;184:2297-304.

35. Yang H, Li N, Song LN, et al. Activation of NOD1 by DAP contributes to myocardial ischemia/reperfusion injury via multiple signaling pathways. Apoptosis 2015;20:51222.

36. Cartwright N, Murch O, McMaster SK, et al. Selective NOD1 agonists cause shock and organ injury/dysfunction in vivo. Am J Respir Crit Care Med 2007;175:595-603.

37. Takahashi T, Kamimura T, Ichida F. Ultrastructural findings on polymorphonuclear leucocyte infiltration and acute hepatocellular damage in alcoholic hepatitis. Liver $1987 ; 7: 347-58$

38. Taieb J, Delarche C, Paradis V, et al. Polymorphonuclear neutrophils are a source of hepatocyte growth factor in patients with severe alcoholic hepatitis. J Hepatol $2002 ; 36: 342-8$.

39. Lazaro R, Wu R, Lee S, et al. Osteopontin deficiency does not prevent but promotes alcoholic neutrophilic hepatitis in mice. Hepatology 2015;61:129-40.

40. Park JK, Utsumi T, Seo YE, et al. Cellular distribution of injected PLGAnanoparticles in the liver. Nanomedicine 2016;12:1365-74. 
41. Zhang Y, Sophocleous AM, Schwendeman SP. Inhibition of peptide acylation in PLGA microspheres with water-soluble divalent cationic salts. Pharm Res 2009;26:1986-94.

42. Qi F, Wu J, Hao D, et al. Comparative studies on the influences of primary emulsion preparation on properties of uniform-sized exenatide-loaded PLGA microspheres. Pharm Res 2014;31:1566-74.

43. Rawat A, Bhardwaj U, Burgess DJ. Comparison of in vitro-in vivo release of Risperdal((R)) Consta((R)) microspheres. Int J Pharm 2012;434:115-21. 


\section{FIGURE LEGENDS}

Figure 1: NOD1 activation induced liver expression of adhesion molecules under physiological condition

A: Representative Western-blot of livers extracted from C57BL/6 mice treated with vehicle or NOD1 agonist (FK565). ICAM-1, VCAM-1, P-selectin and E-selectin (from right to left panel) protein expression were compared to $\beta$-actin. Lower panel histograms represent ratio of band intensity of the adhesion molecules compared to $\beta$-actin. Results are expressed as relative units (RU). Statistical test: Mann Whitney, significance is defined by $\mathrm{p}<0.05$. Statistical significance is indicated.

B: Representative ICAM-1 immunostainings of livers extracted from mice only treated with $\underline{\text { vehicle (left panel) or NOD1 agonist (iE-DAP) (right panel). Negative controls are included }}$ (upper right corner). Livers from vehicle-treated mice displayed significant staining on sinusoids. After NOD1 agonist treatment, sinusoidal staining was reinforced and a marked staining was observed on hepatocytes.

Figure 2: NOD1 activation induced liver expression of adhesion molecules after liver IR

A: Representative H\&E histological picture of livers extracted from C57BL/6 mice that $\underline{\text { underwent ischemia/reperfusion (IR) and were treated with vehicle (IR+Vehicle, Left panel) }}$ or NOD1 agonist (iE-DAP) (IR+NOD1 agonist, right panel). Dashed lines circle areas of liver injury.

B: Histograms representing liver PMN count and relative expression of liver KC mRNA 4, 8 and 20 hours (hrs) after reperfusion in C57BL/6 mice treated with vehicle (IR+Vehicle, white bars) or NOD1 agonist (IR+NOD1 agonist, black bars). Results are expressed as mean \pm SEM. 
$\underline{\text { Statistical test: Mann Whitney, significance is defined by } p<0.05 \text {. Statistical significances are }}$ indicated.

C: Kinetic determination of transaminase AST (left panel) and ALT (right panel) in C57BL/6 mice serum 4,8 and 20 hours after reperfusion and treatment with vehicle (grey line) or NOD1 agonist (black line). Results are expressed as mean \pm SEM. Statistical test: Mann Whitney, significance is defined by $p<0.05$. Statistical significances are indicated.

D: histograms representing early (4 hours after reperfusion) liver expression of Icam-1 and $\underline{\text { Vcam-1 mRNA in C57BL/6 mice treated with vehicle (IR+Vehicle, white bars) or NOD1 }}$ agonist (IR+NOD1 agonist, black bars). Results are expressed as mean \pm SEM. Statistical $\underline{\text { significances are indicated. Statistical test: Mann Whitney, significance is defined by } p<0.05}$

E: Representative Western-blot of liver extracted 20hours after reperfusion from C57BL/6 mice treated with vehicle (IR+Vehicle) or NOD1 agonist (IR+NOD1 agonist). ICAM-1, $\underline{\text { VCAM-1, P-selectin and E-selectin (from right to left panel) protein expressions were }}$ compared to $\beta$-actin. Lower panel histograms represent the ratio of band intensity of the adhesion molecules compared to $\beta$-actin. Results are expressed as relative units (RU). $\underline{\text { Statistical test: Mann Whitney, significance is defined by } p<0.05 \text {. Statistical significance is }}$ indicated.

Figure 3: NOD1 activation induces hepatocyte expression of ICAM-1 and VCAM-1.

A: mRNA expression of Icam-1, Vcam-1, E-selectin and PECAM (from upper to lower panel) in HUVEC, Statistical test: Mann Whitney, significance is defined by $\mathrm{p}<0.05$.

B-C: mRNA expression of Icam-1 (upper panel) and Vcam-1 (lower panel) in primary hepatocytes isolated from Nod1 KO mice (C) or their wild-type littermates (Nod1 WT mice, B). Cells were treated with vehicle or NOD1 agonist (FK565) and/or TNF $50 \mathrm{ng} / \mathrm{ml}$ and IFN $\gamma$ 
$100 \mathrm{ng} / \mathrm{ml}$ (TNF/IFN $\gamma$ ). Results are expressed as relative units (RU). Statistical significance was indicated. Statistical test : Mann Whitney, significance is defined by $\mathrm{p}<0.05$

D-E: Representative western-blots for quantification of ICAM-1 (upper blot) and VCAM-1 (lower blot) protein expression that were compared to $\beta$-actin. Primary hepatocytes isolated from Nod1 KO mice (D) or their wild-type littermates (Nod1 WT mice, C), were treated with vehicle or a NOD1 agonist (FK565) and/or TNF 50 ng/ml and IFN $\gamma 100 \mathrm{ng} / \mathrm{ml}$ (TNF/IFN $\gamma$ ). Results are expressed as relative units (RU). Statistical test : Mann Whitney, significance is defined by $\mathrm{p}<0.05$. Statistical significance is indicated.

Figure 4: During ischemia reperfusion, NOD1 pathway induced hepatocyte expression of adhesion molecules.

A-B: Ex-vivo mRNA expression of Icam-1 (upper panel) and Vcam-1 (lower panel) in primary hepatocytes isolated from Nod1 KO mice (B) or their wild-type littermates (Nod1 WT mice, A). Mice were treated or not with NOD1 agonist (FK565) after sham surgery or ischemia/reperfusion (isch/reperf). Results are expressed as relative units (RU). Statistical test: Mann Whitney, significance is defined by $p<0.05$. Statistical significance is indicated.

C-D: Representative western-blots for quantification of ICAM-1 (upper blot) and VCAM-1 (lower blot) protein expression that were compared to $\beta$-actin in ex vivo primary hepatocytes. Nod1 KO mice (D) or their wild-type littermates (Nod1 WT mice, C), were treated or not with a NOD1 agonist (FK565) after sham surgery or ischemia/reperfusion (isch/reperf). Ratios of band intensity are indicated as relative units (RU).

Figure 5: NOD1 pathway activation leads to hepatocyte lysis by neutrophils. 
Co-culture experiments to assess hepatocyte (Hepato) and neutrophil (PMN) interaction and cell lysis. A: Representative cytology formed by electronic microscopy of the interaction between neutrophils (PMN) and hepatocytes (Hep). B: Primary hepatocytes were isolated from wild-type mice (Hepato WT) treated or/not with a NOD1 agonist (FK565). Then hepatocytes were co-cultured (or not) with PMN harvested from wild type mice. Dosage of LDH in co-culture supernatants (relative unit, RU). C: Primary hepatocytes were isolated from Nod1 KO mice (Hepato KO) treated or/not with a NOD1 agonist (FK565). Then hepatocytes were co-cultured (or not) with PMN harvested from wild type mice. Dosage of LDH in co-culture supernatants (relative unit, RU). Statistical test: Mann Whitney, significance is defined by $\mathrm{p}<0.05$

\section{Figure 6: NOD1 signaling and ICAM-1 promoter}

A-B: Ratio of band intensity of phospho MAPkinases compared to total MAPkinases from western-blots performed on liver extracts from C57BL/6 mice 0, 10, 30, 60 minutes after treatment with a NOD1 agonist (iE-DAP). Mean \pm SD are represented $(n=6)$ and statistical significance is indicated as $*$ for $\mathrm{p}<0.05$ as compared to baseline (time point $0 \mathrm{~min}$ ). Lower panel corresponds to representative Western-blot. A: Liver ERK (p42/p44) activation. B: Liver p38 activation. Statistical test: Mann Whitney, significance is defined by $p<0.05$.

C-D: Histograms for mRNA expression of Icam-1 (C) and Vcam-1 (D) in hepatocytes treated or not with a NOD1 agonist (iE-DAP) and with an ERK inhibitor (U0126, 10 $\mu \mathrm{M})$ and/or a p38 inhibitor (SB203580, 10 $\mu \mathrm{M})$. Results are expressed as relative units (RU, Mean \pm SEM, $\mathrm{n}=5-10)$. Statistical significance is indicated as $*$ for $\mathrm{p}<0.05$ or $* *$ for $\mathrm{p}<0.01$. Untreated control (white bar) was compared to positive control (NOD1 agonist treatment, black bar). Inhibitor treated cells (grey bars) were compared to positive control (black bar). Statistical test: Mann Whitney, significance is defined by $\mathrm{p}<0.05$. 
E: Schematic diagram representing $838 \mathrm{bp}$ of human ICAM-1 promoter been cloned in the reporter vector, pGL4. NF- $\mathrm{kB}$ response element (red boxes) and AP-1 (blue boxes) response elements are indicated. Transcription starts are represented as $(\mapsto)$ and ATG is indicated as a purple box. F: CV-1 cells are transfected or not with human a NOD1 expression vector (phNOD1) and transfected or not with a reporter vector containing human ICAM-1 promoter (hICAM prom). Cells with different transfection conditions are treated or not with a NOD1 agonist (C12-iE-DAP). The human ICAM-1 promoter activity is evaluated using luminescence and quantified as relative light units (RLU). Statistical significance is indicated as *. Statistical test: Mann Whitney, significance is defined by $p<0.05$.

\section{Figure 7: Use of antagonists to target NOD1 in liver.}

C57BL/6 mice received an iv injection of empty (np Empty) or ALINO73-loaded nanoparticles (np ALINO) with a dose corresponding to $5 \mathrm{mg} / \mathrm{kg}$ of ALINO73. $\mathrm{n}=13$ in each group. Six hours later mice underwent $60 \mathrm{~min}$ of ischemia and 20 hours of reperfusion.

A-B: Image of mouse liver histology (H\&E staining) following IR injury. C: Liver from mouse that underwent IR and treated with empty PLGA nanoparticles (IR + np Empty) display large injury areas (surrounded by dotted lines). D: Liver from mouse that underwent IR and treated with ALINO73-loaded nanoparticles (IR + np ALINO). Injury areas are obviously more limited.

C: Quantification of liver necrotic areas from mouse that underwent IR (Liver IR) and treated with empty PLGA nanoparticles (np Empty) and from mouse that underwent IR and treated with ALINO73-loaded nanoparticles (np ALINO). Results are expressed in percentage of necrotic surface among total liver surface as Mean \pm SEM. Statistical test: Mann Whitney, significance is defined by $\mathrm{p}<0.05$. 
D: Serum ALT expressed as international units/liter (IU/L) from mouse that underwent IR (Liver IR) and treated with empty PLGA nanoparticles (np Empty) and from mouse that underwent IR and treated with ALINO73-loaded nanoparticles (np ALINO). Statistical significances are indicated. Statistical test: Mann Whitney, significance is defined by $p<0.05$. E: Histogram representing liver PMN count 20 hours after reperfusion (Liver IR) in mice $\underline{\text { treated with empty PLGA nanoparticles (np Empty) and mice treated with ALINO73-loaded }}$ nanoparticles (np ALINO). PMN infiltrating liver parenchyma (Extravasated PMN, white $\underline{\text { bars) or in centrolobular veins (PMN in vessels, grey bars) were distinguished. Results are }}$ expressed in number of PMN per field of necrosis (magnification $\mathrm{x} 400$ ) as mean \pm SEM. $\underline{\text { Statistical test: Mann Whitney, significance is defined by } p<0.05 \text {. Statistical significances are }}$ indicated.

F: Histogram representing liver expression of KC mRNA 20 hours after reperfusion in mice $\underline{\text { treated with empty PLGA nanoparticles (np Empty) and mice treated with ALINO73-loaded }}$ nanoparticles (np ALINO). Results are expressed in relative units (RU) as mean \pm SEM. $\underline{\text { Statistical test: Mann Whitney, significance is defined by } p<0.05 \text {. Statistical significances are }}$ indicated.

G: Representative ICAM-1 immunostainings of livers harvested from mice that underwent $\underline{\text { liver IR and were treated with empty nanoparticles (np Empty, left panel) or with np ALINO }}$ (right panel) 


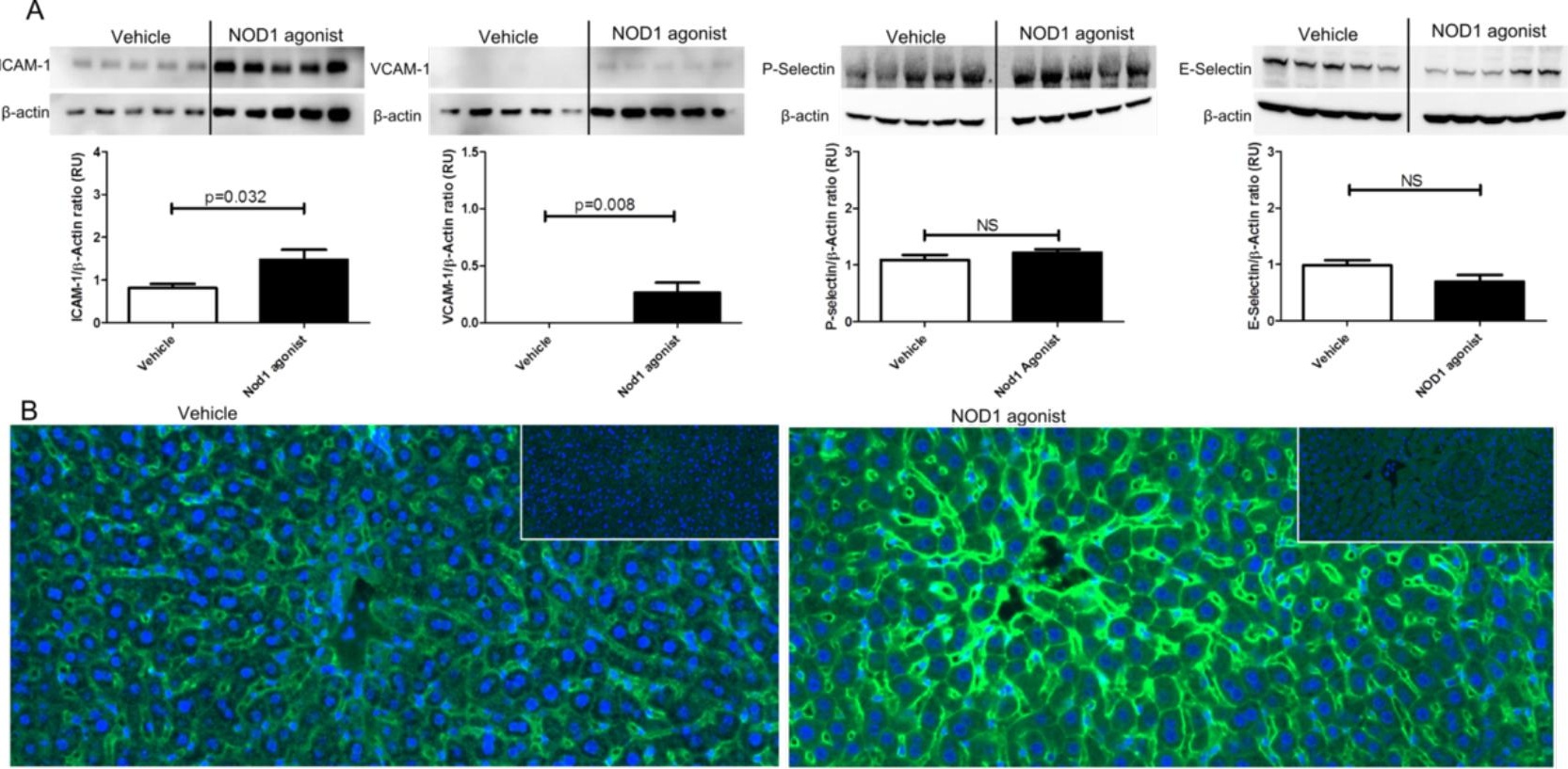




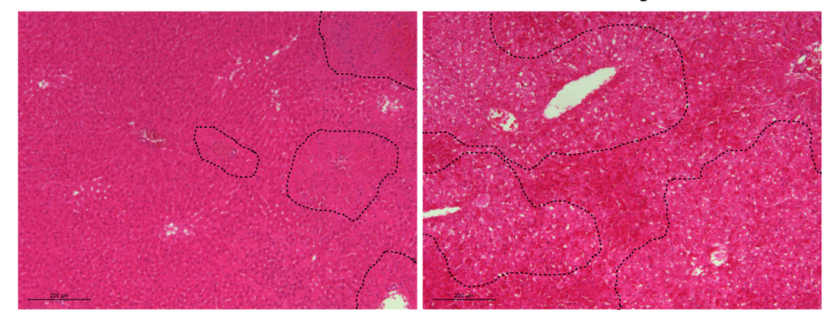

C

AST

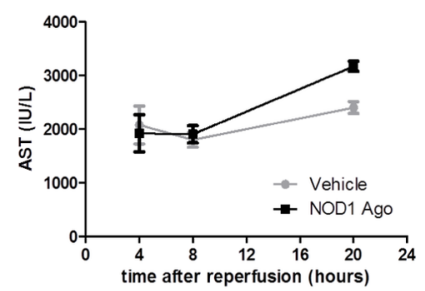

$\mathrm{E}$

E
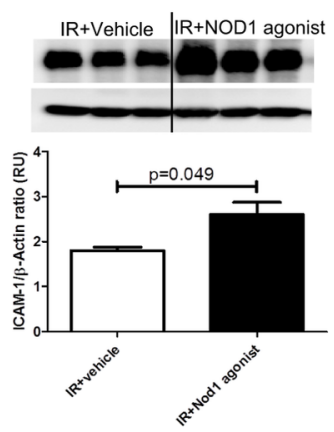

ALT

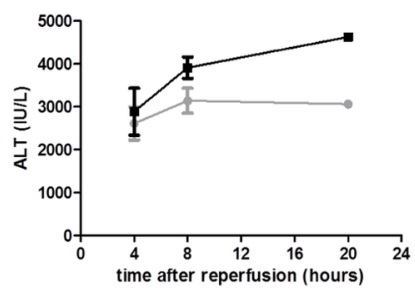

\begin{tabular}{l|l}
$\mathrm{IR}+$ Vehicle & $\mathrm{IR}+\mathrm{NOD} 1$ agonist
\end{tabular}
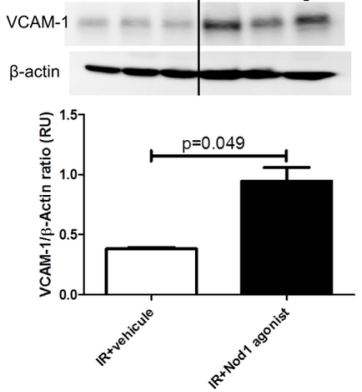

PMN count

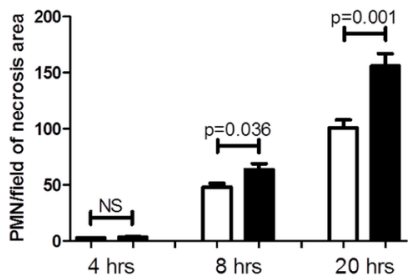

D
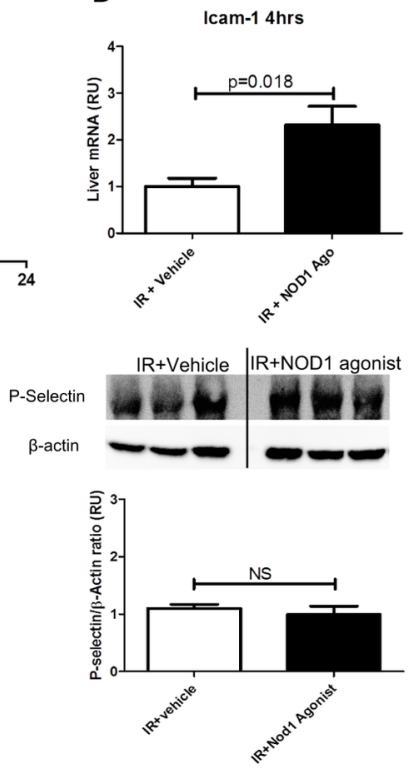

KC

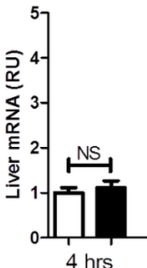

$\stackrel{N S}{\longleftarrow}$

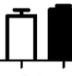

$8 \mathrm{hrs}$
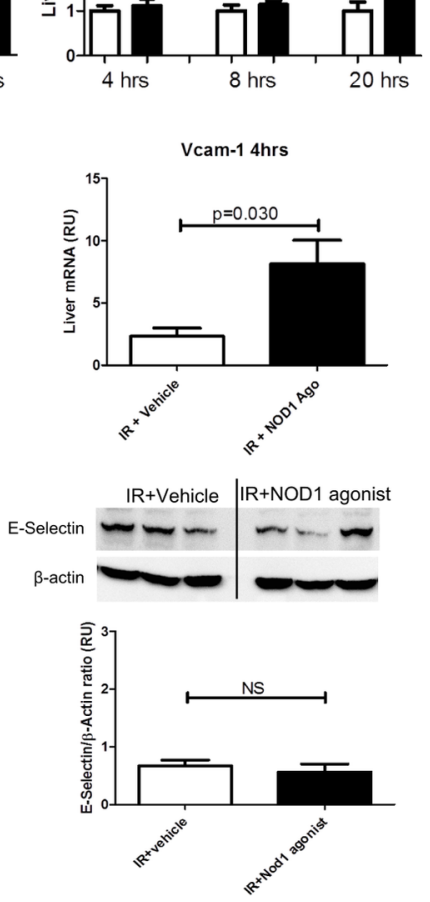


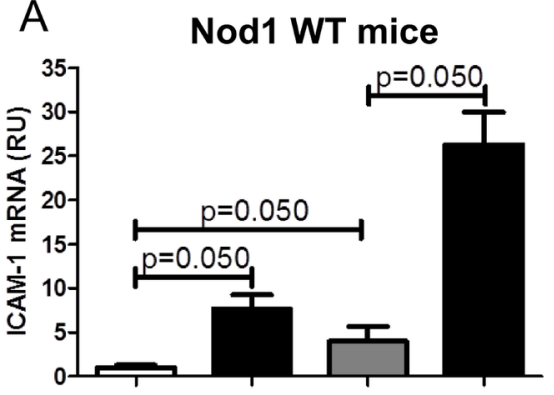

B Nod1 KO mice

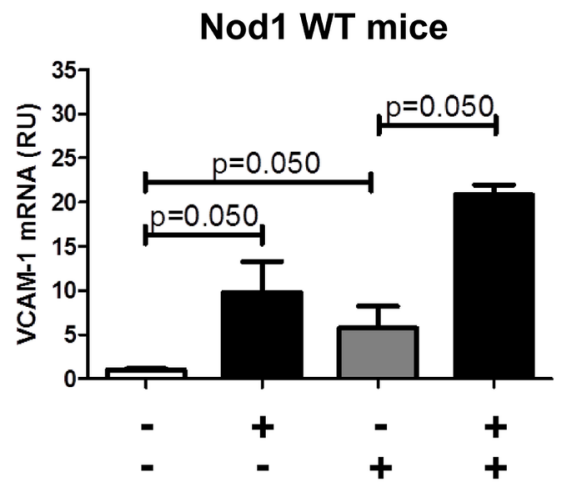

C Nod1 WT mice
$1.1 \quad 7.8$
$2.3 \quad 40.8$

D

ICAM-1

$\beta$-actin
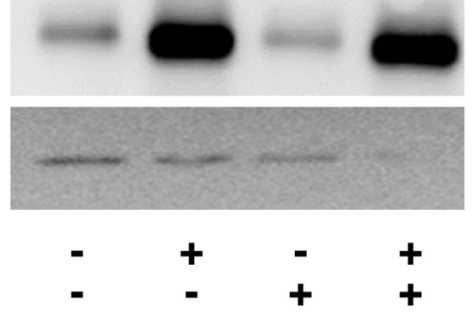

NOD1 agonist isch/reperf
0.1
0.4
0.2
10.9

Nod1 KO mice

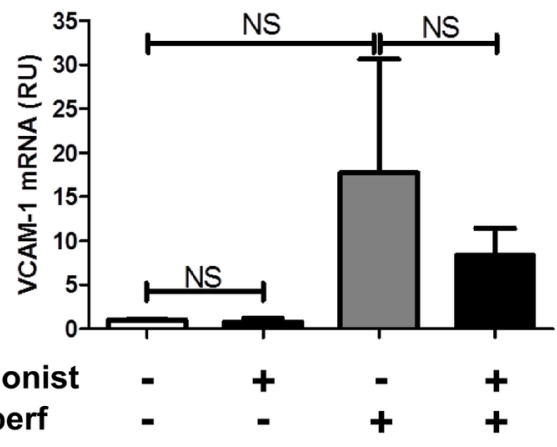
$0.9 \quad 1.1$
0.9
0.9

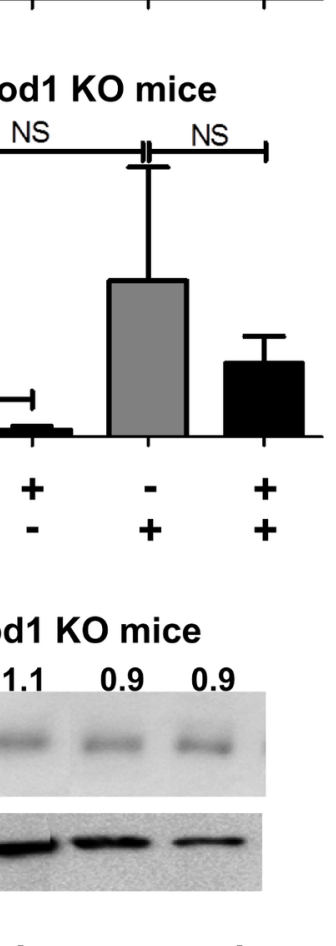

$\begin{array}{lll}+ & - & + \\ - & + & +\end{array}$

$\begin{array}{llll}0.9 & 0.4 & 1.5 & 1.2\end{array}$

VCAM-1 

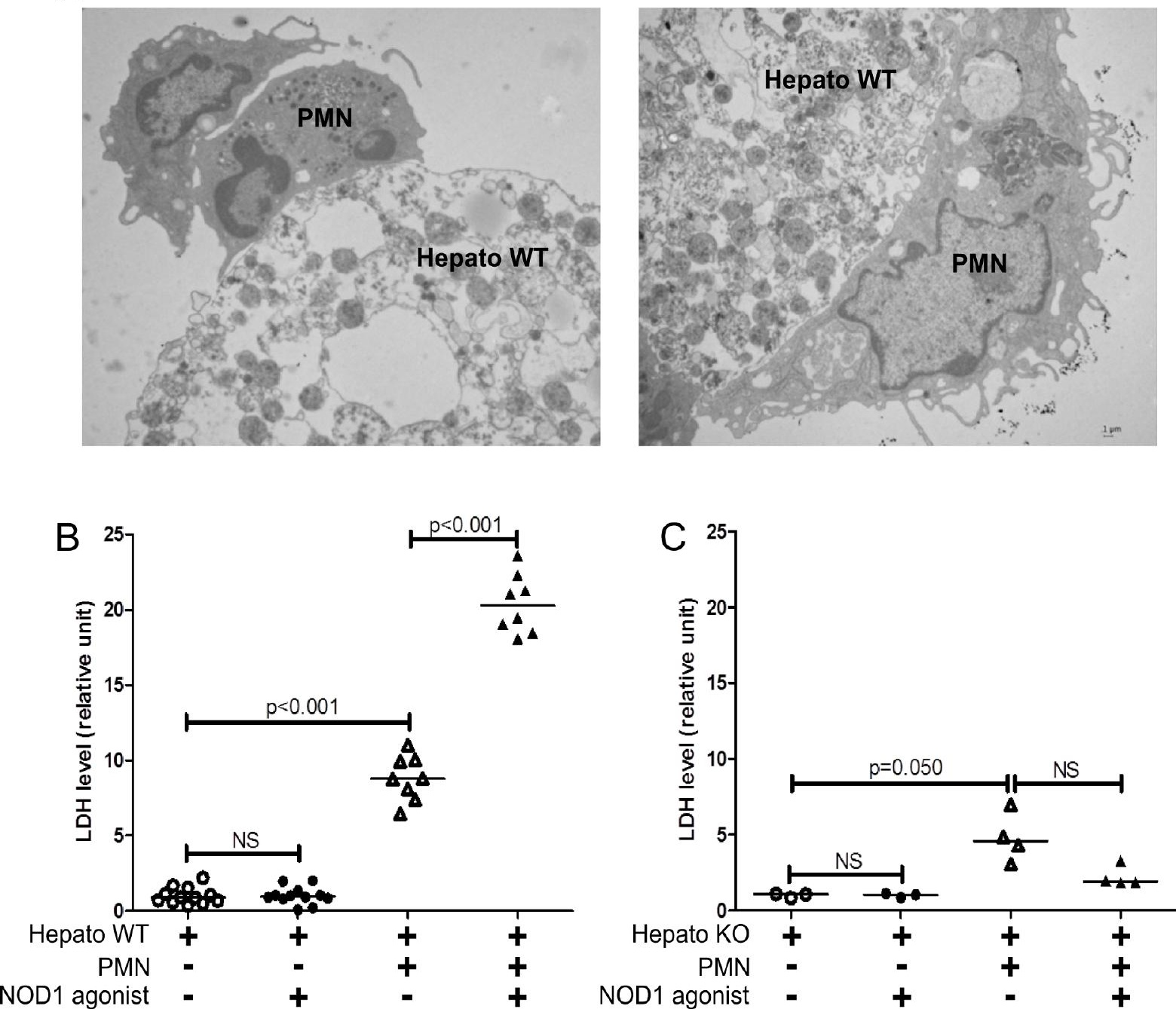


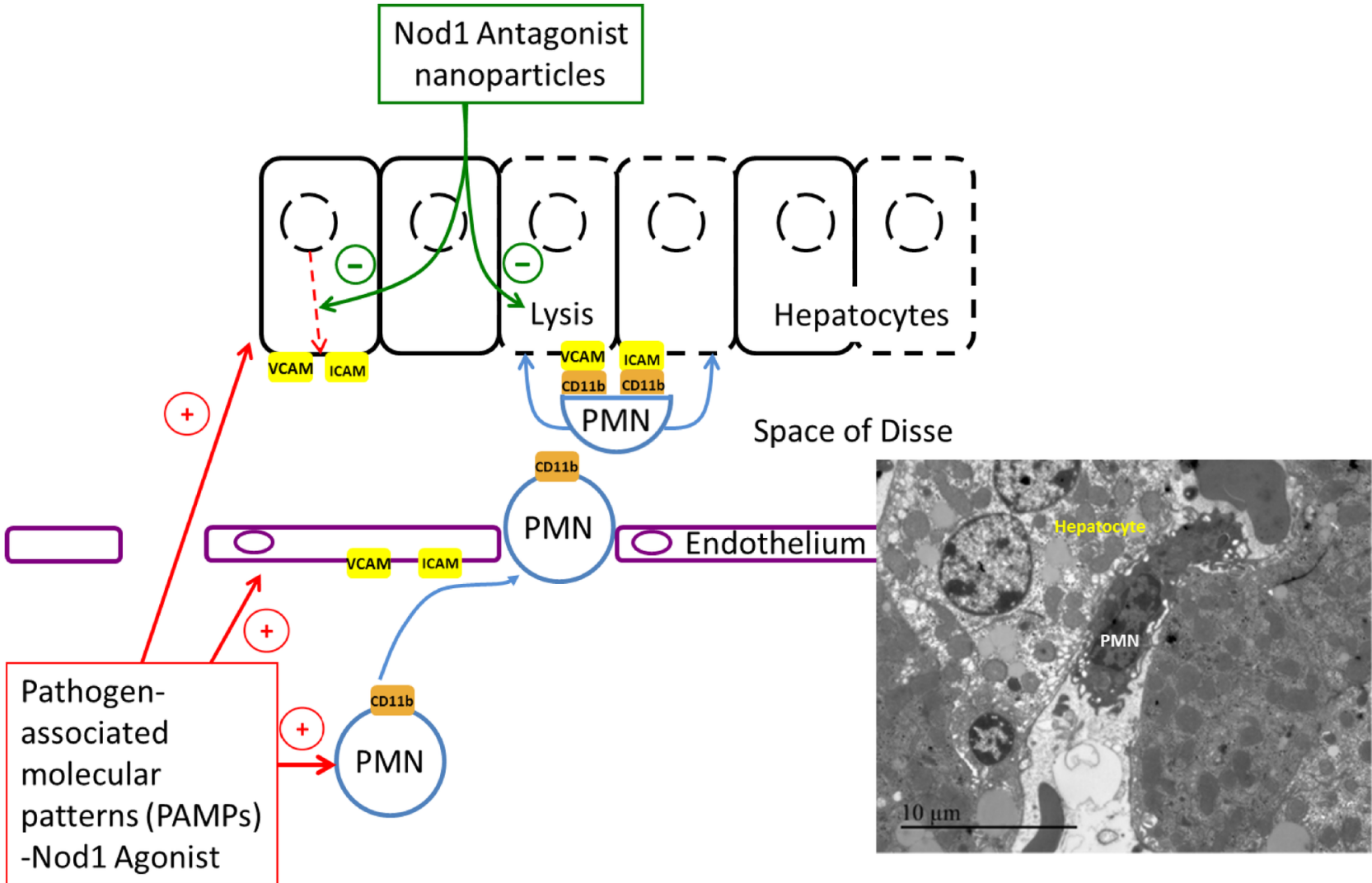

